\title{
Process Realization of Preparing the Plane Transient Diode
}

\author{
An Yu, Wu Youlin
}

Department of Physics and Electronic Engineering, Zunyi Normal College, Zunyi, Guizhou, 563002

Keywords: Cleaning, oxidation, photolithography, diffusion, epitaxy

\begin{abstract}
The process flows of preparing the plane transient diode have been described in this paper. The process purpose, the process conditions, the process steps and the process methods of each part in flows have been also analyzed in detail. At the same time, the process technology of the qualified transient diodes has been also experimentally discussed, which has provided the foundation for the production.
\end{abstract}

\section{The fabrication process of the plane transient diode is shown in Fig}

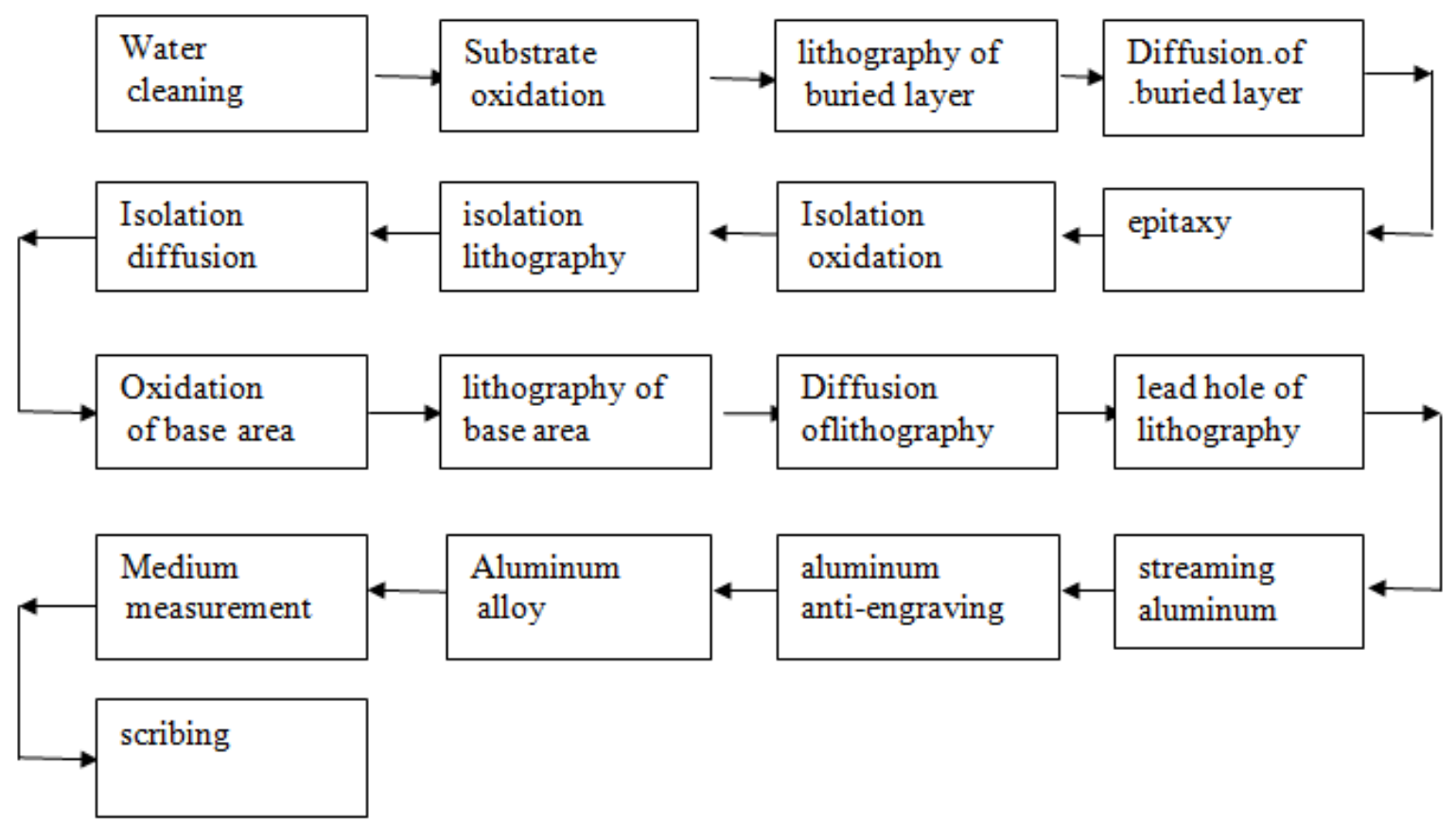

Fig 1 Fabrication process chart

The specific steps can be shown as follows:

Silicon wafer cleaning, oxidizing, photoetching, diffusion, epitaxyThe wafer cleaning. The PFD of wafer cleaning can be shown as follows:

The wafer to be cleaned--- washing the ionized water--- boiling the $1^{\#}$ liquid--- water filling--rinse of hydrofluoric acid solution--- water filling--- boiling the $2^{\#}$ liquid--- water filling--- drying up

The positive side and the negative side of the original wafer should be wiped in one direction through the cotton ball and the acetone. The negative side should be firstly wiped. Then the positive side should be wiped. Afterwards, the wafer should be placed into the clean baskets. According to the process flow diagram, we sh0ould firstly configure the $1^{\#}$ liquid, the hydrofluoric acid solution and the $2^{\#}$ liquid. 
(1) The configuration of $1^{\#}$ liquid

The configuration ratio of $1^{\#}$ liquid is: ammonia: hydrogen peroxide: ionized water= 1: $2: 5$ (volume ratio). According to the specific needs of experiment, the total volume of the solution should be $880 \mathrm{~mL}$, then $\mathrm{NH}_{4} \mathrm{OH}: \mathrm{H}_{2} \mathrm{O}_{2}: \mathrm{H}_{2} \mathrm{O}=110: 220: 550$.

(2) The configuration of hydrofluoric acid solution

Similarly, the total volume of the solution should be $880 \mathrm{~mL}$, then $\mathrm{HF}: \mathrm{H}_{2} \mathrm{O}=80: 800$ (volume ratio).

(3) The configuration of $2^{\#}$ liquid

The configuration ratio of $2^{\#}$ liquid is: hydrochloric: hydrogen peroxide: ionized water=1:2: 8 (volume ratio). The total volume of the solution should be $880 \mathrm{~mL}$, then $\mathrm{HCL}: \mathrm{H}_{2} \mathrm{O}_{2}: \mathrm{H}_{2} \mathrm{O}=80: 160$ : 640.

The specific cleaning steps can be shown as follows:

According to the above proportion, the $1^{\#}$ liquid should be prepared. Then the basket equipped with wafer should be put into the $1^{\#}$ liquid. When it is boiled, the residue should be discarded. It should be washed by the ionized water for $10-15 \mathrm{~min}$.

It is necessary to configure the hydrofluoric acid solution (the volume ratio is 10:1). The wafer which is boiled in $1^{\#}$ liquid should be placed into the configured hydrofluoric acid solution. When the negative side of wafer is dehydrated, the basket should be washed by the ionized water for $10-15 \mathrm{~min}$.

It is required to configure the $2^{\#}$ liquid. The washed wafer should be boiled for $3-5 \mathrm{~min}$ in the $2^{\#}$ liquid. Afterwards, the residue should be drained and it should be washed by ionized water for $10-15 \mathrm{~min}$. at the same time, it should be also placed into the "B expansion" oven to bake for $20 \mathrm{~min}$. The wafer cleaning process will run through the whole plane process experiment.

The substrate oxidation. The purpose of substrate oxidation is to provide the shelter for the lithography of buried layer. It is required to adopt the dry oxygen + wet oxygen + dry oxygen oxidation method. Then the film of obtained silica will be uniform and compact, the thickness will be uniform and the surface quality will be well. The PFD can be shown as follows:

Warming--- the silicon wafer is shipped--- the valve is opened when the furnace temperature is $900^{\circ} \mathrm{C}$--- the silicon wafer is put into the furnace--- the wet oxygen valve is turned on--- the nitrogen valve and the oxygen valve are turned on--- the oxygen valve is turned off and the nitrogen valve is turned on--- the temperature is reduced to $900^{\circ} \mathrm{C}---$ the silicon wafer is taken out

The dried silicon wafer should be installed on the boat face to face and back to back. The positioning surface should be upward. At the same time, it is also necessary to install the monitor film. The room temperature should be up to $900^{\circ} \mathrm{C}$ after $45 \mathrm{~min}$. When the temperature is $900^{\circ} \mathrm{C}$, the silicon wafer should be placed into the quartz boat after half an hour in constant temperature. The boat should be pushed into the constant temperature zone in oxygen (the flow of $\mathrm{O}_{2}$ is $1-1.5 \mathrm{~L} / \mathrm{min}$ ). The furnace temperature should be risen from $900^{\circ} \mathrm{C}$ to $1180^{\circ} \mathrm{C}$ with the speed of $300^{\circ} \mathrm{C} / \mathrm{h}$ after $53 \mathrm{~min}$. When the temperature is $1180^{\circ} \mathrm{C}$, it is required to keep the constant temperature for $70 \mathrm{~min}$. During this period, the dry oxygen valve should be turned on for $10 \mathrm{~min}$. Afterwards, the oxygen should be changed into wet oxygen(the oxygen should be through the boiling water). The flow of oxygen is 0.8 $1.5 \mathrm{~L} / \mathrm{min}$. After 50 minutes, the mixture of nitrogen and oxygen should be turned on for $10 \mathrm{~min}$. Then the oxygen valve should be turned off and the nitrogen valve should be turned on for $45 \mathrm{~min}$. When the furnace temperature is decreased from $1180^{\circ} \mathrm{C}$ to $900^{\circ} \mathrm{C}$, the silicon wafer should be taken out.

Afterwards, it is required to observe the quality of oxide layer on the surface of silicon wafer. The dark blue on the surface of silicon wafer is uniformly distributed, which indicates that the quality of oxide layer is well and it can be sent to the next process for experiment.

The lithography of buried layer. The purpose of the lithography of buried layer is to provide the needed window for the diffusion of buried layer. It is required to employ the contact exposure method. The negative photoresist is used to conduct the selective corrosion for the silica layer. Then the graphics corresponding to the lithographic plate on the silica will be obtained.

The process diagram of lithography can be shown as follows:

Spin coating--- prebaking--- exposure--- developing and fixing--- checking--- hardening--corrosion--- removing of photoresist--- self-checking 
Spin coating: it is required to start the spin coating machine to place the silicon wafer into the center of vacuum suction of spin coating machine. The frontage of wafer should be upward. Afterwards, the vacuum suction piece should be connected and the ear washing ball is employed to clean the surface of silicon surface. The speed of spin coating machined is set as $4000 \mathrm{r} / \mathrm{min}$ and the spin coating time is $50 \mathrm{~s}$. The plastic head dropper should drop certain photoresist to the surface of silicon wafer. When the spin coating machine is turned off and the silicon wafer is stopped rotating, the vacuum should be immediately closed. Then the silicon wafer should will be removed from chuck and placed into the silicon frame.

Pre-baking: when all the silicon wafers are uniformly glued, they will be placed into the front oven. The temperature of oven should be set as $90^{\circ} \mathrm{C}$ and the time of prebaking is $30 \mathrm{~min}$. The silicon wafer should be taken out after 30min, which will be sent to the lithography machine for exposure after being cooled.

Exposure: it is required to open the lithography vacuum pump and the nitrogen valve. The pressure should be adjusted to $0.3 \mathrm{Mpa}$ and the lithography plate should be installed on the exposure machine. The exposure time is $10-30$ s for the wafer aligner.

Developing and fixing: it is necessary to put the exposed silicon wafer into the center of vacuum suction of spin coating machine. The wafer should be upward. Afterwards, it is required to start the spin coating and the time is $60 \pm 10 \mathrm{~s}$. The rotating speed is $4300 \mathrm{r} / \mathrm{min}$. The plastic head dropper should drop certain developing solution to the surface of silicon wafer. When the silicon wafer stops rotating, it is required to reset the fixing time, which is $30 \pm 10 \mathrm{~s}$. Afterwards, the spin coating machine is started and the fixing solution is dropped on the surface of silicon wafer. Then the silicon wafer should be removed from the chuck and placed into the silicon frame. Finally, it is required to check the results after development.

Hardening: the inspected silicon wafer should be put into the hardening film. The temperature of oven is $160^{\circ} \mathrm{C}$. The silicon wafer should be taken out after $30 \mathrm{~min}$ and it should be naturally cooled.

Chemical corrosion: the surface of silicon wafer after hardening has been formed the graphics on board from the photoresist. Afterwards, it is required to use the etching solution prepared by HF to corrode the excess silica. The temperature of corrosive environment is $36-39^{\circ} \mathrm{C}$ and the etching time is about $3-5 \mathrm{~m} 30 \mathrm{~s}$.

Removing of photoresist: the silicon wafer should be soaked into the stripping solution and it shouldn't be hearted until there is the white smoke. Afterwards, it is needed to remove the silicon wafer from the etching solution after five minutes. The silicon wafer is required to be washed by the ionized water for 30 minutes in the beaker. Finally, it should be dried and checked.

The diffusion of buried layer zone. The silicon wafer engraved with the graphics of buried layer region should use $\mathrm{Sb}_{2} \mathrm{O}_{3}$ : $\mathrm{Sio}_{2}=1: 3$ source to conduct the diffusion of buried layer zone through the prewashing before the diffusion. Considering the thickness of buried layer is 4 microns, the specific operation steps can be shown as follows:

The furnace temperature should be risen from $900^{\circ} \mathrm{C}$ to $1200^{\circ} \mathrm{C}$ and the gas flow is $\mathrm{N}_{2} 1.5 \mathrm{~L} / \mathrm{min}$.

The installed Si should be placed into the mouth of furnace and preheated for five minutes. Then it is required to be pulled into the constant temperature zone.

When the temperature is $1200^{\circ} \mathrm{C}$, it is required to diffuse for about three hours $\left(\mathrm{O}_{2} 2 \mathrm{~L} / \mathrm{min}\right.$ should be added for 150 minutes after adding $1.5 \mathrm{~L} / \mathrm{min}_{2}$ ).

The temperature should be decreased to $800^{\circ} \mathrm{C}$. The quartz box should be pulled to the mouth of furnace with the five minutes casting speed. Afterwards, it should be cooled for ten minutes (at least). Then the Si wafer should be taken out.

The epitaxy after removing the oxide layer. After the diffusion of buried layer, the N-type impurity region with a certain thickness will be generated in the base film. At the same time, there is also an oxide layer on the surface of base film, which is not demanded in the later process. Therefore, we can adopt the hydrofluoric acid solution to corrode it. Afterwards, the epitaxy can be conducted. According to the thickness of epitaxial layer, the epitaxy can be achieved through controlling the time and the temperature. The impurity of epitaxy still belongs to $\mathrm{N}$ type. The selected materials are $\mathrm{SiCl}_{4}$ and $\mathrm{PCl}_{3}$. The steps can be shown as follows: 
1. Opening the cover of quartz tube (the masks and the gloves must be required).

2. Taking out the graphite and installing the clean test pieces.

3. Pushing the graphite to the constant temperature zone of epitaxial furnace.

4. Covering the pipe of quartz tube.

5. Turning on the hydrogen valve for 20 or $25 \mathrm{~L} / \mathrm{min}$.

6. The corrosion time is set as 2 minutes, the time of casting gas is 2 minutes, the growth time is 5 minutes and the heating time is 2 minutes. The epitaxial growth rate is controlled around $1 \mu \mathrm{m} / \mathrm{min}$. Generally speaking, the flow of $\mathrm{HCl}$ is $0.3 \mathrm{~L} / \mathrm{min}$, the flow of $\mathrm{PCl}_{3}$ is $50 \mathrm{~mL} / \mathrm{min}$ and the $\mathrm{SiCl}_{4}$ is $3 \mathrm{~L} / \mathrm{min}$.

7. Connecting the voltage on the control box.

8. Operating the voltage regulator knob on the control box and slowly heating up.

9. Heating up for 3-5 minutes (at this time, the positive current is $1.2 \mathrm{~A}$ and the grid current is around $450 \mathrm{~mA}$ ). When the temperature is constant, it is required to start the switch on the control panel.

10. The execution of program is completed and it is necessary to cool for 3-5 minutes. Afterwards, the high voltage should be turned off.

11. It is required to cool for five minutes. Then it is needed to open the cover of quartz tube to take out the wafer.

Oxidizing isolation, photoetching, diffusionThe isolation oxidation. The purpose of isolation oxidation is to provide the shelter for the isolation lithography. The oxidation steps are similar to the substrate oxidation. The typical technology conditions are:

The oven temperature: $\mathrm{T}=1100 \pm 1^{\circ} \mathrm{C}$. The temperature in the oven and out of the oven is $900^{\circ} \mathrm{C}$.

Process: (1)the time of turning on the oxygen valve is: $10 \mathrm{~min} \pm 20$ s (it is required to start timing when the temperature is constant)

(2) The time of turning on the wet oxygen valve: $(120-180) \mathrm{min} \pm 1 \mathrm{~min}$

(3) The time of turning on $\mathrm{N}_{2}$ valve: $30 \mathrm{~min} \pm 10 \mathrm{~min}$

The isolation lithography. The purpose of isolation lithography is to open the isolation diffusion window. The steps and the process technology conditions are same as the lithography of buried layer. The isolation diffusion. The purpose of isolation diffusion is to form the isolation junction through expanding into the P-type impurity in the isolation window. The diffusion time is determined by the depth of isolation junction. As the depth of isolation junction is $6 \mu \mathrm{m}$, so it is required to employ the two-step diffusion method. The diffusion source adopts the boron microcrystalline glass. The process can be shown as:

It is required to deposit $\mathrm{B}_{2} \mathrm{O}_{3}$ for $45-50 \mathrm{~min}$ in $1130 \pm 1^{\circ} \mathrm{C}$.

It is necessary to turn on the $\mathrm{N}_{2}$ valve and conduct the main extension for one hour in $1270^{\circ} \mathrm{C} \pm 1$ ${ }^{\circ} \mathrm{C}$. In this process, the surface concentration has reached the saturation solubility. In order to inhibit the excessive $\mathrm{N}_{\mathrm{s}}$ and remove the BSG, it is required to change into the oxygen atmosphere, namely, the dry oxygen valve should be turned on for 1.5 hours. At the same time, the boron should be further diffused to the P-type substrate. The temperature in the oven and out of oven is $900^{\circ} \mathrm{C}$

Base oxidizing, photoetching, diffusionThe oxidation of base area. The purpose of oxidation of base area is to provide the shelter for the lithography of base area. The oxidation steps are similar to the steps of substrate oxidation. The typical technology conditions are:

The oven temperature: $\mathrm{T}=1100 \pm 1^{\circ} \mathrm{C}$. The temperature in the oven and out of the oven is $900^{\circ} \mathrm{C}$.

Process: (1)The time of turning on the dry oxygen valve is: $10 \mathrm{~min}$

(2) The time of turning on the wet oxygen valve: $70 \mathrm{~min}$

(3) The time of turning on dry $\mathrm{O}_{2}$ valve: $30 \mathrm{~min}$

The thickness of oxide layer: $0.5 \pm 0.005 \mu \mathrm{m}$. 
The lithography of base area. The purpose of lithography of base area is to open the diffusion window in the silicon wafer. The steps are same as the lithography of buried layer. The typical technology conditions are:

The speed of spin coating is $5000 \mathrm{r} / \mathrm{min}$ and the time is $25 \pm 5 \mathrm{~s}$.

The temperature of prebaking is 90 degrees and the time of prebaking is $30 \mathrm{~min}$.

The exposure time is $8-20 \mathrm{~s}$.

The time of developing is $60 \pm 10 \mathrm{~s}$ and the speed is $4300 \mathrm{r} / \mathrm{min}$. The time of fixing is $30 \pm 10 \mathrm{~s}$.

The oven temperature when hardening is 150 degrees and the time of hardening is $30 \mathrm{~min}$.

The diffusion of base area. The diffusion of base area refers that the P-type impurity is diffused into the epitaxial layer. Then the contact of growth P-type area and the extended $\mathrm{N}$ area will form the PN junction. The diffusion time is determined by the impurity concentration and the depth of junction, etc. The selected impurity is B source. The deposition should be firstly conducted. The temperature of deposition is $\mathrm{T}=960^{\circ} \mathrm{C} \pm 1^{\circ} \mathrm{C}$ and the time is about $20-30 \mathrm{~min}$. The diffusion should be conducted after the deposition and the temperature is $\mathrm{T}=\left(1150^{\circ} \mathrm{C}-1180{ }^{\circ} \mathrm{C}\right) \pm 1^{\circ} \mathrm{C}$. Within this temperature range, according to the relationship diagram ${ }^{[1]} 2$ of base area, temperature and diffusion time, the diffusion time is probably estimated as 60 minutes when the depth of junction is $3.2 \mu \mathrm{m}$.

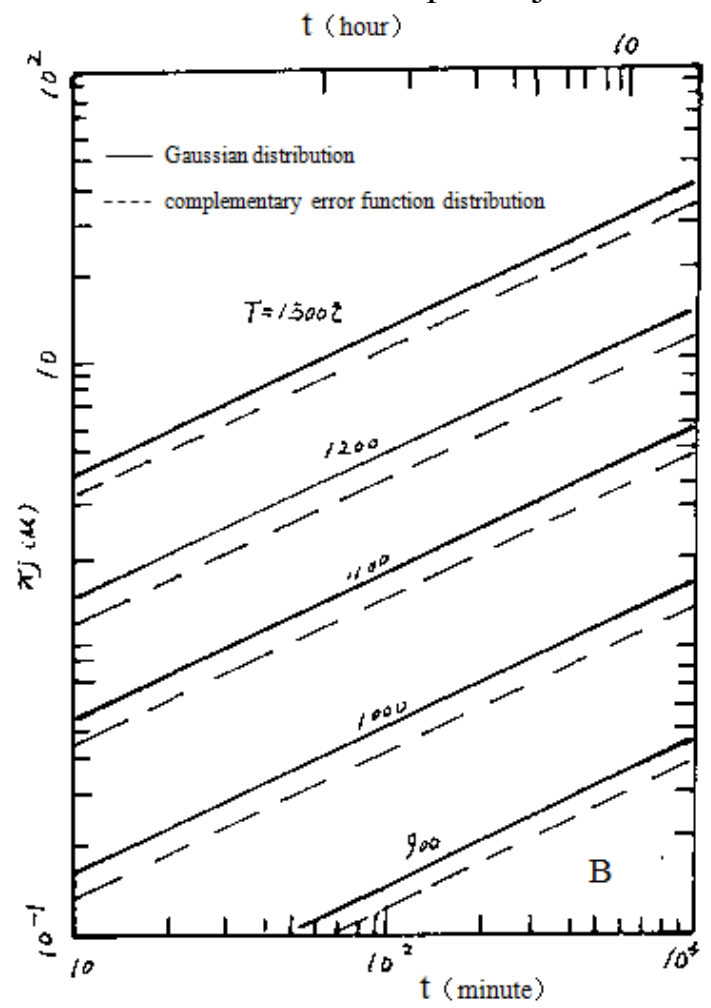

Figure 2 The curve of the relationship between the boron junction depth, $\mathrm{T}$ and $\mathrm{t}$

Then the diffusion time is $t_{\text {total }}=t_{\mathrm{o}_{2 \mathrm{~d}}}+\mathrm{t}_{\mathrm{o} 2 \mathrm{w}_{\square}}+\mathrm{t}_{\mathrm{o}_{2 \mathrm{~d}}}=60 \pm 1(\mathrm{~min})$. The time of turning on the oxygen valve is about 40 minutes and the time of dry oxygen is determined by the test pieces.

Photoetching hole, aluminum film evaporating, aluminum etching, aluminum alloyingThe lead hole of lithography. After the diffusion of base area, it is necessary to open the lead holes in the silicon wafer. The purpose is to introduce the electrodes of polarization of PN junction. The typical lithography technology conditions are:

The speed of spin coating is $5000 \mathrm{r} / \mathrm{min}$ and the time is $25 \pm 5 \mathrm{~s}$.

The temperature of prebaking is 90 degrees and the time of prebaking is $30 \mathrm{~min}$.

The exposure time is $8-20 \mathrm{~s}$.

The time of developing is $60 \pm 10 \mathrm{~s}$ and the speed is $4300 \mathrm{r} / \mathrm{min}$. The time of fixing is $30 \pm 10 \mathrm{~s}$.

The oven temperature when hardening is 150 degrees and the time of hardening is $30 \mathrm{~min}$. 
The streaming aluminum. The streaming aluminum refers that there is a layer of aluminum deposited on the surface of silicon wafer.

The aluminum anti-engraving. The aluminum anti-engraving refers that it is required to selectively carve the places where the wiring and the bonding are required on the surface of silicon wafer. The typical technology conditions are:

The speed of spin coating is $4000 \mathrm{r} / \mathrm{min}$ and the time is $30 \pm 5 \mathrm{~s}$.

The temperature of prebaking is 90 degrees and the time of prebaking is $30 \mathrm{~min}$.

The exposure time is $10-20 \mathrm{~s}$.

The time of developing is $60 \pm 10 \mathrm{~s}$ and the speed is $4300 \mathrm{r} / \mathrm{min}$. The time of fixing is $30 \pm 10 \mathrm{~s}$.

The oven temperature when hardening is 160 degrees and the time of hardening is $30 \mathrm{~min}$.

The aluminum. When the aluminum is evaporated onto the surface of silicon wafer and the electrode pattern with a certain shape is formed after the lithography, it is required to go through the alloy process. The purpose of aluminum is to form the ohmic contact with low resistance and increase the adhesion between the aluminum and the silica films. Then the weld will be firmly fixed on the chip when the hot pressing and the ultrasound key are connected. The alloying process refers that the anti-carved silicon wafer is placed into the vacuum room or the furnace tube with the protection of nitrogen. It should be heated to a certain temperature and the temperature should be kept constant for a period of time. And the ohmic contact with low resistance will be obtained after being cooled. At last, people can make scribing after CHB test

\section{Acknowledgments}

This work is supported partly by the key support discipline of Guizhou province No. ([2011] 275).

\section{References}

[1] [U.S.] H.F. Wolf. Translated by Semiconductor Plant in Tianjin. Data Sheet of Silicon Semiconductor Process[M]. Beijing: National Defense Industry Press, 1975, 163.

[2] Catalog and Application Guide of Transient Voltage Suppression Diode Product[Z]. Guiyang: State-owned No. 873 Factory, 2001.

[3] Generic File of Bipolar Silicon Planar Power Transistor Chip Manufacturing Process(trial); Key Laboratory of Micro-Nano Electronics and Software Technology in Guizhou.

[4] [U.S.] Shi Min. Translated by Huang Zhengang. Semiconductor Device Physics[M]. Beijing: Electronic Industry Press, 1987.

[5] [U.S.] Donald A. Neamen. Translated by Zhao Yiqiang, Yao Suying, Xie Xiaodong et al. Semiconductor Physics and Devices[M]. Beijing: Electronic Industry Press.

[6] Huang Hanyao, Li Naiping, Sunqing et al. Principles of Semiconductor Device Technology[M]. Beijing: National Defense Industry Press.

[7] Zhao Baojing. Design and Manufacture of High-power Transistors[M]. Beijing: Science Press, 1978.

[8] The professional Technical Teaching Material Writing Group of Electronic Industry Semiconductor. The Semiconductor Device Technology[M]. Shanghai: Shanghai Science and Technology Literature Press, 1984.

[9] [U.S.] Michael Quirk, Julian Serda. Translated by Han Zhengsheng. Semiconductor Manufacturing Technology[M]. Beijing: Electronic Industry Press, 2005. 